\title{
Macroalgas em nascentes e arredores de riachos na região noroeste do Estado de São Paulo
}

\author{
Jeferson Luizi Pereira ${ }^{1,2}$ e Luis Henrique Zanini Branco ${ }^{1}$
}

Recebido: 09.11.2009; aceito: 01.07.2010

\begin{abstract}
Macroalgae in springs and surrounding streams of northwestern region of São Paulo State). Macroalgae are typically benthic organisms that a mature, discreet and recognizable naked eye thallus, and microscopic identification is necessary. Several studies have been carried out aiming to the taxonomic knowledge in Brazilian streams, however works dealing with algae from springs are rare. Monthly sampling were carried out from February to September/2007 in springs and the surrounding (humid soil near the springs) of four streams in the northwestern region of São Paulo State. The floristic survey resulted in the identification of 13 species, being $54 \%$ of taxa belong Cyanophyta and $46 \%$ to Chlorophyta. Cylindrospermum gorakhpurense, Mougeotia gotlandica, Spirogyra gallica and S. taftiana were recorded for the first time to Brazil. The aquatic flora differed from the regional records due to presence of organisms of Zygnemataceae family (Chlorophyta) in reproductive stage. This type of habitat revealed to be very favorable to the Zygnemataceae reproduction.
\end{abstract}

Key words: flora, Spirogyra, subaerial, Zygnemataceae

RESUMO - (Macroalgas em nascentes e arredores de riachos na região noroeste do Estado de São Paulo). Macroalgas são organismos tipicamente bentônicos, constituídos por um talo maduro, discreto e reconhecível a olho nu, cuja identificação microscópica é necessária. Diversos estudos foram realizados visando o conhecimento taxonômico em riachos brasileiros, no entanto, são raros os trabalhos que abordam as algas que se desenvolvem nas nascentes destes riachos. De fevereiro a setembro/2007 coletas mensais foram realizadas em nascentes e em suas imediações (solos úmidos dos afloramentos d'água) de quatro riachos na região noroeste do estado de São Paulo. O levantamento taxonômico resultou na identificação de 13 espécies, sendo 54\% dos táxons pertencentes a Cyanophyta e $46 \%$ a Chlorophyta. Cylindrospermum gorakhpurense, Mougeotia gotlandica, Spirogyra gallica e S. taftiana são registradas pela primeira vez para o Brasil. A flora aquática encontrada diferiu da regional devido à presença de organismos da família Zygnemataceae (Chlorophyta) em estágio reprodutivo. Este tipo de hábitat mostrou-se especialmente favorável à reprodução das Zygnemataceae.

Palavras-chave: flora, Spirogyra, subaérea, Zygnemataceae

\section{Introdução}

Em ambientes aquáticos é possível distinguir as microalgas e as macroalgas. Estas últimas são organismos tipicamente bentônicos, geralmente reconhecíveis a olho nu. Contudo, a identificação microscópica é muitas vezes necessária e, frequentemente, as microalgas encontram-se associadas aos seus talos (Sheath \& Cole 1992). Alguns estudos, como os de Branco \& Necchi Júnior (1996), Necchi Júnior et al. (1997), Branco et al. (1999) e Vieira Júnior et al. (2002) foram realizados visando o conhecimento da flora de macroalgas em riachos do Estado de São Paulo. No entanto, estes estudos não abordaram as algas que se desenvolvem nas numerosas nascentes que alimentam estes riachos.

De acordo com Round (1983), as nascentes frias suportam uma flora algal em qualquer substrato sólido, arenoso, siltoso ou vivo que se encontre em suas águas, e as espécies, em sua maioria, também são encontradas em outros hábitats, em particular os de água corrente, pois uma nascente é quase sempre o início de um ambiente lótico. Geralmente, por representarem sistemas geograficamente isolados, as nascentes podem apresentar numerosos táxons endêmicos, como, por exemplo, de macroalgas (e.g. Vis \& Sheath 1996). Contudo, de acordo com

\footnotetext{
1. Universidade Estadual Paulista (UNESP), Departamento de Zoologia e Botânica, Rua Cristóvão Colombo, 2265, 15054-000 São José do Rio Preto, SP, Brasil

2. Autor para correspondência: pereira.j1@terra.com.br
} 
Necchi Júnior (1992), esta flora não é tão diferente daquela encontrada em ambientes de águas correntes.

Estudos ficológicos envolvendo nascentes são escassos mundialmente, sendo que, os poucos existentes se concentram na América do Norte (Whitford 1956, Leake \& Leake 1961, Vis \& Sheath 1996, Sherwood \& Sheath 1999, Sherwood et al. 2000) e Europa (Żelazna-Wieczorek \& Ziułkiewicz 2004), além de enfatizarem principalmente as diatomáceas. No Brasil, estes estudos estão limitados quase inteiramente a Kleerekoper (1944) que mencionou a ocorrência de diatomáceas (Bacillariophyceae) em minas do estado de Minas Gerais e a Necchi Júnior (1992) que verificou a dinâmica da comunidade de macroalgas em uma nascente do estado de São Paulo. Assim, o presente estudo tem por finalidade conhecer a flora de macroalgas que se desenvolve nas nascentes e arredores de alguns riachos da região noroeste do Estado de São Paulo.

\section{Material e métodos}

Coletas aleatórias e mensais foram realizadas de fevereiro a setembro de 2007 em nascentes e imediações (solo úmido dos afloramentos d'água) de quatro riachos na região noroeste do Estado de São Paulo (figura 1): córrego Água Limpa, município de Bady Bassitt (204'3"S e 4928'10"W), córrego Castores, município de Onda Verde (20³8'29'S e 49 $\left.18^{\prime} 19^{\prime \prime} \mathrm{W}\right)$, córrego do Cocho, município de Cedral (2058'36"S e 49²0'23”W) e córrego do Trigo, município de São José do Rio Preto (2050'56"S e 4922'11"W).

Alguns parâmetros ambientais foram avaliados em cada um dos locais amostrados. Dentre estes se analisou a condutividade, oxigênio dissolvido, $\mathrm{pH}$, temperatura e turbidez, utilizando-se o controlador de qualidade de água HORIBA U-10; além da profundidade e tipo de substrato.

Amostras dos crescimentos algais, que eram visíveis a olho nu, foram coletadas e fixadas em solução de formaldeído $4 \%$ em frascos de polietileno ou vidro. Em todas as observações e registros foi utilizado um microscópio óptico binocular Jenaval equipado com câmara clara e sistema fotográfico.

\section{Resultados e Discussão}

Todas as nascentes estudadas eram do tipo reocreno, ou seja, a água ao sair forma imediatamente

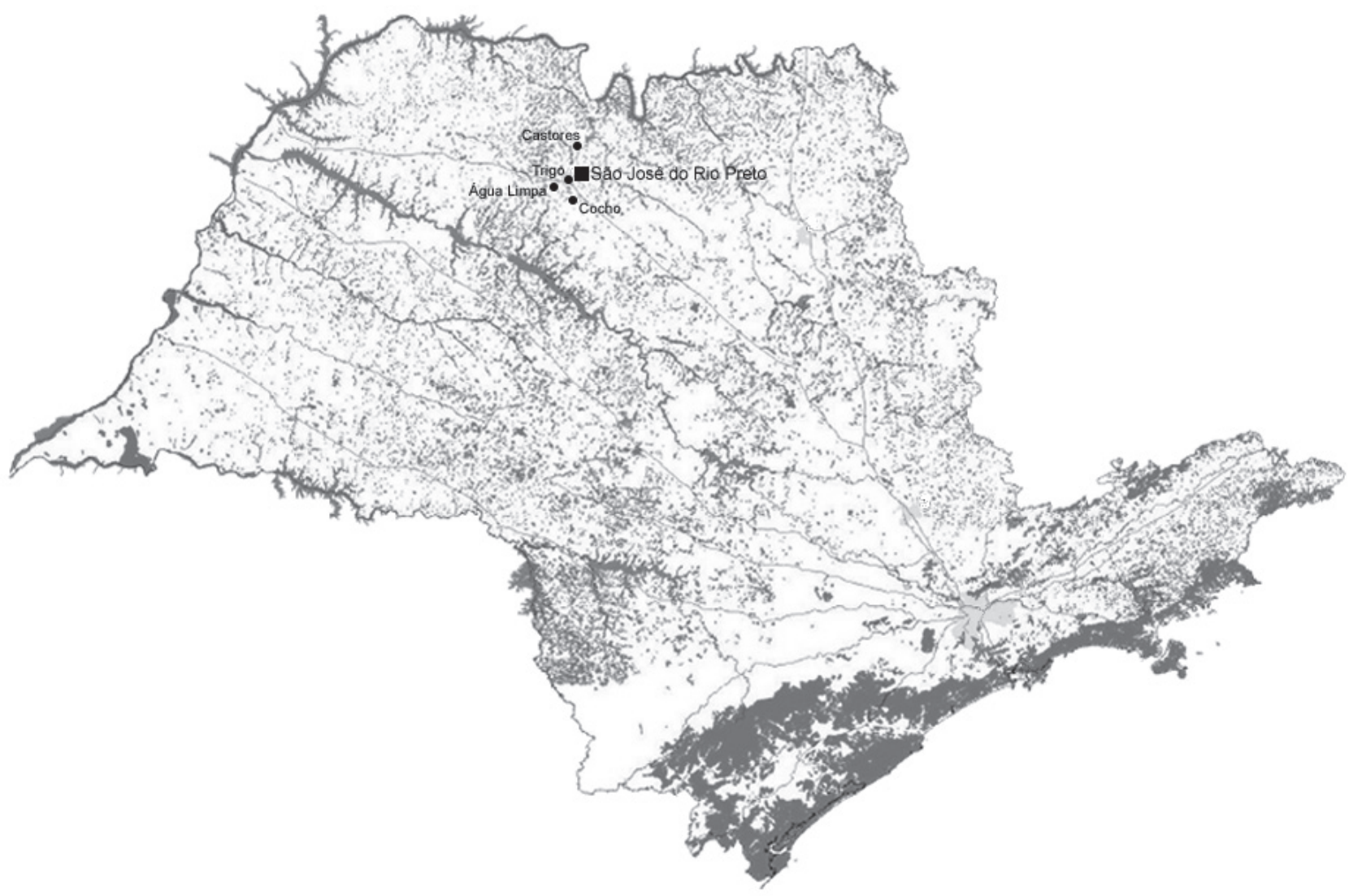

Figura 1. Localização na região noroeste do Estado de São Paulo dos riachos com as nascentes estudadas.

Figure 1. Localization of the studied springs streams in the northwest region of the São Paulo State. 
um riacho com maior ou menor correnteza na nascente (Kleerekoper 1944). O ambiente caracteriza-se por apresentar vegetação marginal variando de gramíneas a briófitas, sombreamento moderado, condutividade elétrica de 5,4-19,6 $\mu \mathrm{S} \mathrm{cm} \mathrm{cm}^{-1}$, oxigênio dissolvido de 4,7-6,0 $\mathrm{mg} \mathrm{L}^{-1}$, $\mathrm{pH}$ entre 6,5-7,1, temperatura de $18,9-24,2^{\circ} \mathrm{C}$, turbidez de 5,9-11 UNT, profundidade de $2-4 \mathrm{~cm}$ e substrato essencialmente arenoso. Estes dados não diferiram de estudos anteriores feitos em riachos da região (Necchi Júnior et al. 1994, 1997, Branco \& Necchi Júnior 1998, Vieira Júnior et al. 2002) ou de outra nascente (Necchi Júnior 1992).

Apenas representantes das divisões Cyanophyta e Chlorophyta foram encontrados, totalizando 13 espécies identificadas. O grupo mais bem representado foi Cyanophyta compreendendo 54\% dos táxons (sete espécies), enquanto Chlorophyta apresentou $46 \%$ (seis espécies).

Cyanophyta

Cylindrospermum gorakhpurense R.N. Singh, Proc. Ind. Acad. Sci. B, 9: 65. 1939.

Figuras 2-3

Massa prostrada gelatinosa, verde-azulada; tricomas flexuosos, isolados em uma matriz mucilaginosa incolor; células cilíndricas, 3,7 $\mu \mathrm{m}$ diâm., (6,2-)7,5-10(-11,2) $\mu \mathrm{m}$ compr.; heterócitos terminais, levemente cônicos, 5-6,2 $\mu \mathrm{m}$ diâm., (7,5-)8,7-10(-11,2) $\mu \mathrm{m}$ compr.; acinetos simples, elipsoidal, episporo incolor, ornamentado com projeções laterais em forma de agulha, (7,5-)8,712,5(15) $\mu \mathrm{m}$ diâm., 20-25(-30) $\mu \mathrm{m}$ compr. O material analisado é relativamente maior que o encontrado por Desikachary (1959), contudo concorda com este autor quanto ao hábitat.

Hábitat: solo úmido entre gramíneas em mina d'água no córrego do Cocho.

Material examinado: BRASIL. SÃo PAUlo: Cedral, córrego do Cocho, 19-V-2007, J.L. Pereira s.n. (SJRP29708).

Distribuição no Brasil: primeira citação para o Brasil.

Nostoc muscorum Agardh ex Bornet \& Flahault, Ann. Sci. Nat. Bot., ser. 7, 7: 200. 1888.

Figuras 4-5

Colônias macroscópicas, irregularmente arranjadas, amareladas; bainha amarelada; tricomas frouxamente emaranhados; células cilíndricas ou levemente elípticas, 2,5-3,7 $\mu$ m diâm., (3,7-)5-6,2(7,5) $\mu \mathrm{m}$ compr.; heterócitos terminais, arredondados,
3,7-5 $\mu \mathrm{m}$ diâm., 3,7-5(-6,2) $\mu \mathrm{m}$ compr.; acinetos oblongos, às vezes em série, distantes dos heterócitos, 5-6,2 $\mu \mathrm{m}$ diâm., 7,5-8,7 $\mu \mathrm{m}$ compr.

Hábitat: solo úmido da margem de afloramento d'água no córrego do Cocho.

Material examinado: BRASIL. São PAULO: Cedral, córrego do Cocho, 24-III-2007, J.L. Pereira s.n. (SJRP29703).

Distribuição no Brasil: Estados do Rio Grande do Sul (Yunes et al. 1990, Yunes et al. 1994, Werner 2002), Santa Catarina (Moebius 1895) e São Paulo (Borge 1918, Drouet 1938, Oliveira 1978, Azevedo 1991, Sant'Anna 1991, Sant'Anna et al. 1991, Azevedo et al. 1996).

Nostoc piscinale Kützing ex Bornet \& Flahault, Ann.

Sci. Nat. Bot., ser. 7, 7: 194. 1888.

Figura 6-7

Colônias macroscópicas, membranáceas, esverdeadas; bainha amarelada; tricomas emaranhados; células arredondadas, 3,7-5 $\mu \mathrm{m}$ diâm., 3,7-5(-6,2) $\mu \mathrm{m}$ compr.; heterócitos terminais ou intercalar, arredondados, 6,2-7,5 $\mu \mathrm{m}$ diâm., 7,5(8,7) $\mu \mathrm{m}$ compr.; acinetos esféricos ou levemente alongados, às vezes em série, distantes dos heterócitos, 6-6,5 $\mu \mathrm{m}$ diâm., 6-7,5 $\mu \mathrm{m}$ compr.

Hábitat: solo úmido na margem de mina d'água no córrego do Trigo.

Material examinado: BRASIL. SÃo Paulo: São José do Rio Preto, córrego do Trigo, 24-IX-2007, J.L. Pereira s.n. (SJRP29706).

Distribuição no Brasil: Estados do Amazonas (Drouet 1938), Ceará (Drouet 1938), Rio de Janeiro (Oliveira \& Cardoso 1982), São Paulo (Wittrock \& Nordstedt 1880, Edwall 1896, Borge 1918, Drouet 1938, Sant'Anna 1991, Azevedo et al. 1996).

Phormidium ornatum (Kützing ex Gomont) Anagnostidis \& Komárek, Arch. Hydrobiol. Suppl. Algol. Stud. 80: 406. $1988 \equiv$ Oscillatoria ornata Kütz. ex Gom., Ann. Sci. Nat. Bot., Ser. 7, 16: 214. 1892.

\section{Figura 8}

Massa azul-escura brilhante; tricomas solitários, retos, levemente constritos, atenuados, 8-10 $\mu \mathrm{m}$ diâm., células (3-)4-5 $\mu \mathrm{m}$ compr., (0,3-)0,4-0,6 vez mais longas que largas; conteúdo celular azulado, granulado; septos levemente granulados; célula apical arredondada. As citações na distribuição desta espécie tratam-na como Oscillatoria ornata Kützing ex Gomont. 
Hábitat: submerso em afloramento d'água no córrego do Trigo.

Material examinado: BRASIL. São PaUlo: São José do Rio Preto, córrego do Trigo, 20-VIII-2007, J.L. Pereira s.n. (SJRP29711).

Distribuição no Brasil: Estado do Rio Grande do Sul (Werner \& Rosa 1992), São Paulo (Azevedo et al. 1996, Branco \& Pereira 2002).

Porphyrosiphon notarisii Kützing ex Gomont, Ann.

Sci. Nat. Bot., ser. 7, 15: 331. 1892.

Figura 9

Massa prostrada avermelhada; filamentos frouxamente emaranhados, (17,5-)22,5-35(-40) $\mu \mathrm{m}$ diâm.; bainha espessa, lamelada, acastanhada, avermelhada ou incolor; tricomas retos, levemente constrito ou ausente, não atenuados, 13-15(-17,5) $\mu \mathrm{m}$ diâm.; células 5-7,5 $\mu \mathrm{m}$ compr., $0,3-0,5$ vez mais longas que largas; conteúdo celular azul-esverdeado, não granulado; célula apical truncada.

Hábitat: solo úmido na margem de afloramento d'água no córrego do Cocho.

Material examinado: BRASIL. São PAULO: Cedral, córrego do Cocho, 24-III-2007, J.L. Pereira s.n. (SJRP29705).

Distribuição no Brasil: Estados do Amazonas (Drouet 1938), Ceará (Drouet 1937), Maranhão (Drouet 1937), Pará (Drouet 1938), São Paulo (Borge 1918, Drouet 1938, Sant'Anna et al. 1978, Sant'Anna \& Azevedo 1995, Branco et al. 2009).

Scytonema coactile Montagne ex Bornet \& Flahault, Ann. Sci. Nat. Bot., ser. 7, 5: 90. 1887.

Figura 10

Tufos azul-escuros; filamentos emaranhados, (15-)17-20 $\mu \mathrm{m}$ diâm.; falsas ramificações duplas ou simples; bainha espessa, hialina ou levemente amarelada, homogênea ou lamelada; tricomas retos, constritos geralmente no ápice, não atenuados, 10$15 \mu \mathrm{m}$ diâm.; células 5-10(-15) $\mu \mathrm{m}$ compr., 0,4-1($1,5)$ vezes mais longas que largas; conteúdo celular azul-esverdeado, levemente granulado; heterócitos intercalares, pouco frequentes, subquadrados a mais longos do que largos, 10-12(-15) $\mu \mathrm{m}$ diâm., 7-10(-12) $\mu \mathrm{m}$ compr.; célula apical arredondada a hemisférica. Hábitat: submerso em afloramento d'água no córrego do Cocho.

Material examinado: BRASIL. São Paulo: Cedral, córrego do Cocho, 19-V-2007, J.L. Pereira s.n. (SJRP29709).

Distribuição no Brasil: Estados da Paraíba (Drouet
1938), Rio Grande do Sul (Franceschini 1983), São Paulo (Drouet 1938, Sant'Anna et al. 1978, 1983, Sant'Anna 1988).

Scytonema fritschii Ghose, J. Linn. Soc. Bot. Lond. 46: 342. 1923.

Figura 11

Massa verde-azulada; filamentos emaranhados, 15-20 $\mu \mathrm{m}$ diâm.; falsas ramificações duplas ou simples; bainha espessa, hialina ou levemente amarelada, lamelada; tricomas retos, constritos geralmente no ápice, não atenuados, 9-11 $\mu \mathrm{m}$ diâm.; células (4-)5-12(-15) $\mu \mathrm{m}$ compr., 0,4-1,5($1,6)$ vezes mais longas que largas; conteúdo celular azul-esverdeado, levemente granulado; heterócitos intercalares, frequentes, quadrados e raramente mais longos do que largos, $10 \mu \mathrm{m}$ diâm., 10-11 $\mu \mathrm{m}$ compr.; célula apical hemisférica.

Hábitat: submerso em afloramento d'água no córrego do Cocho.

Material examinado: BRASIL. SÃo PAULo: Cedral, córrego do Cocho, 19-V-2007, J.L. Pereira s.n. (SJRP29710).

Distribuição no Brasil: Estado de São Paulo (Sant'Anna 1988).

Chlorophyta

Gloeocystis cf. ampla (Kützing) Lagerheim, Öfvers. K. VetenskAkad. Förh. 40(2): 63. 1883.

Figura 12

Massa gelatinosa verde-clara; colônias constituídas frequentemente por duas células, raramente quatro; células arredondadas a levemente

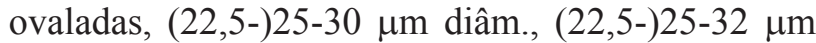
compr.; bainha concêntrica, hialina; cloroplasto cupuliforme, parietal; presença de vacúolos em algumas células. O material analisado difere de Bicudo \& Picelli-Vicentim (1988) quanto ao comprimento e diâmetro das células (13-16 × 12$15 \mu \mathrm{m})$, no entanto, assemelha-se quanto ao hábitat. Contudo, a morfologia desta espécie concorda com a descrita por Guerrero (1948), cujo diâmetro e comprimento celular variam de 18-36 $\mu \mathrm{m}$.

Hábitat: solo úmido entre briófitas em mina d'água no córrego Castores.

Material examinado: BRASIL. SÃo PAUlo: Onda Verde, córrego Castores, 31-IX-2007, J.L. Pereira s.n. (SJRP29712).

Distribuição no Brasil: Estado do Rio de Janeiro (Bicudo \& Picelli-Vicentim 1988). 

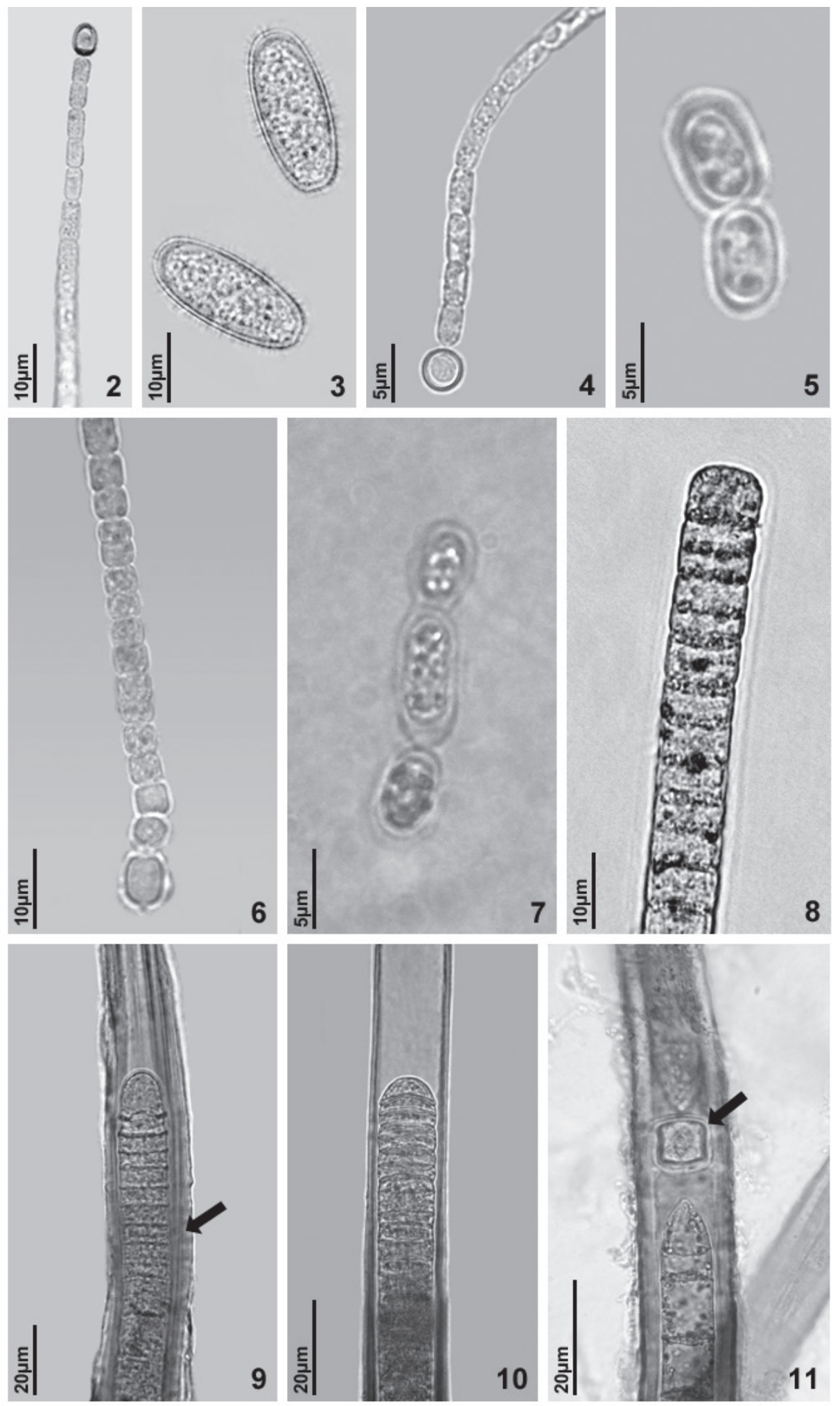

Figuras 2-11. Espécies de macroalgas de nascentes e arredores de riachos na região noroeste do Estado de São Paulo. 2-3. Cylindrospermum gorakhpurense. 2. Parte de filamento com heterócitos. 3. Acinetos soltos. 4-5. Nostoc muscorum. 4. Parte de filamento com heterócito terminal. 5. Acinetos soltos. 6-7. Nostoc piscinale. 6. Parte de filamento com heterócito terminal. 7. Acinetos soltos. 8. Phormidium ornatum, parte de filamento sem bainha. 9. Porphyrosiphon notarisii, parte de filamento com bainha lamelada (seta). 10. Scytonema coactile, parte de filamento com bainha. 11. Scytonema fritschii, parte de filamento com heterócito intercalar (seta).

Figures 2-11. Species of macroalgae in springs and surrounding streams of northwestern region of São Paulo State. 2-3. Cylindrospermum gorakhpurense. 2. Filament part with heterocytes. 3. Unattached akinetes. 4-5. Nostoc muscorum. 4. Filament part with terminal heterocyte. 5. Unattached akinetes. 6-7. Nostoc piscinale. 6. Filament part with terminal heterocyte. 7. Unattached akinetes. 8. Phormidium ornatum, filament part without sheath. 9. Porphyrosiphon notarisii, filament part with lamelated sheath (arrow). 10. Scytonema coactile, filament part with sheath. 11. Scytonema fritschii, filament part with intercalar heterocyte (arrow). 
Mougeotia gotlandica (Cleve) Wittrock, Bih. Kgl. Svensk. Vetensk. Akad. Handl. 1: 39. $1872 \equiv$ Mesocarpus gotlandicus Cleve, Nova Acta Reg. Soc. Sci. Upsali., Ser. 3, 6: 31. 1868.

\section{Figura 13}

Massa filamentosa verde-escura; células vegetativas 9-10 $\mu \mathrm{m}$ diâm., 60-120 $\mu \mathrm{m}$ compr.; cloroplasto com 5-6 pirenóides espalhados; conjugação escalariforme; zigósporos em tubos de conjugação, globosos, 18-25 $\mu \mathrm{m}$ diâm., 18-23 $\mu \mathrm{m}$ compr., marrom, enrugados. Os espécimes analisados apresentam medidas inferiores às descritas por Transeau (1951), Randhawa (1959) e Kadlubowska (1984). Contudo, a presença de zigósporos com paredes enrugadas é uma particularidade deste táxon. Hábitat: submerso em afloramento d'água no córrego do Cocho.

Material examinado: BRASIL. São PAULO: Cedral, córrego do Cocho, 17-II-2007, J.L. Pereira s.n. (SJRP29702).

Distribuição no Brasil: primeira citação para o Brasil.

\section{Oedogonium globosum Nordstedt f. nanyohense} Jao, Sinensia 9(5-6): 263. 1938.

Figura 14

Massa filamentosa verde-amarelada; espécie monóica; células vegetativas cilíndricas, 12-14 $\mu \mathrm{m}$ diâm., 30-40 $\mu \mathrm{m}$ compr.; oogônios globosos a subglobosos, 35-40(-45) $\mu \mathrm{m}$ diâm., 30-45(-55) $\mu \mathrm{m}$ compr., poro superior, parede lisa; oósporos globosos a subglobosos, 25-30(-40) $\mu \mathrm{m}$ diâm., 25-40 $\mu \mathrm{m}$ compr., parede lisa; anterídios 7-8 $\mu \mathrm{m}$ diâm., 4-5 $\mu \mathrm{m}$ compr.

Hábitat: submerso em mina d'água no córrego do Cocho.

Material examinado: BRASIL. São PAulo: Cedral, córrego do Cocho, 19-V-2007, J.L. Pereira s.n. (SJRP29714).

Distribuição no Brasil: Estado de São Paulo (Pereira \& Branco 2006).

Spirogyra gallica Petit, Les Spirogyres des environs de Paris: 23. 1880.

Figuras 15-16

Massa filamentosa verde-brilhante; células vegetativas 50-60 $\mu \mathrm{m}$ diâm., (80-)240-300(-530) $\mu \mathrm{m}$ compr., septos planos; 1 ou 2 cloroplastos, filamentoso achatado, margem espinhosa; conjugação escalariforme, tubos formados por ambos os gametângios, gametângios levemente inflados; zigósporos elipsóides ou ovalados, 45-55(-60) $\mu \mathrm{m}$ diâm., (70-)75-110(-130) ｕm compr.; mesósporo liso, marrom-escuro.

Hábitat: submerso em mina d'água no córrego do Cocho.

Material examinado: BRASIL. SÃo PaUlo: Cedral, córrego do Cocho, 19-V-2007, J.L. Pereira s.n. (SJRP29707).

Distribuição no Brasil: primeira citação para o Brasil.

Spirogyra taftiana Transeau, Ohio Jour. Sci. 44: 243. 1944.

Figuras 17-18

Massa filamentosa verde-fosca; células

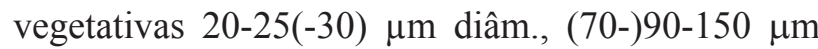
compr., septos planos; 1 cloroplasto, filamentoso achatado, margem espinhosa; conjugação escalariforme, tubos formados por ambos os gametângios, gametângios inflados; células estéreis muitas vezes buliformes; zigósporos elipsóides,

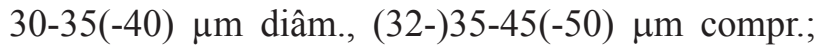
mesósporo estriado, marrom-amarelado.

Hábitat: submerso em afloramento d' água no córrego do Cocho.

Material examinado: BRASIL. São PAULo: Cedral, córrego do Cocho, 24-III-2007, J.L. Pereira s.n. (SJRP29704).

Distribuição no Brasil: primeira citação para o Brasil.

Sirocladium robustum Pereira \& Branco, Hoehnea 34(1): 103. 2007.

Figura 19

Massa filamentosa verde-clara; células vegetativas 65-90 $\mu \mathrm{m}$ diâm., 245-500 $\mu \mathrm{m}$ compr.; 2 cloroplastos, laminar, parietal, com as margens laciniadas, às vezes ramificados, com 5-20 $\mu \mathrm{m}$ diâm. e 15-25 pirenóides dispostos regularmente; conjugação escalariforme, ausência de tubos de conjugação, gametângios de tamanhos diferentes ( $ठ$ menor e q maior). Zigósporos elipsóides ou globosos, 65100(-110) $\mu \mathrm{m}$ diâm., 85-175 $\mu \mathrm{m}$ compr., mesósporo espesso, liso e marrom amarelado.

Hábitat: solo úmido em olho d'água no córrego Água Limpa.

Material examinado: BRASIL. SÃo PAULO: Bady Bassitt, córrego Água Limpa, 24-III-2007, J.L. Pereira s.n. (SJRP29713).

Distribuição no Brasil: Estado de São Paulo (Pereira \& Branco 2007).

Dos 13 táxons amostrados, sete foram encontrados crescendo submersos em nascentes, 

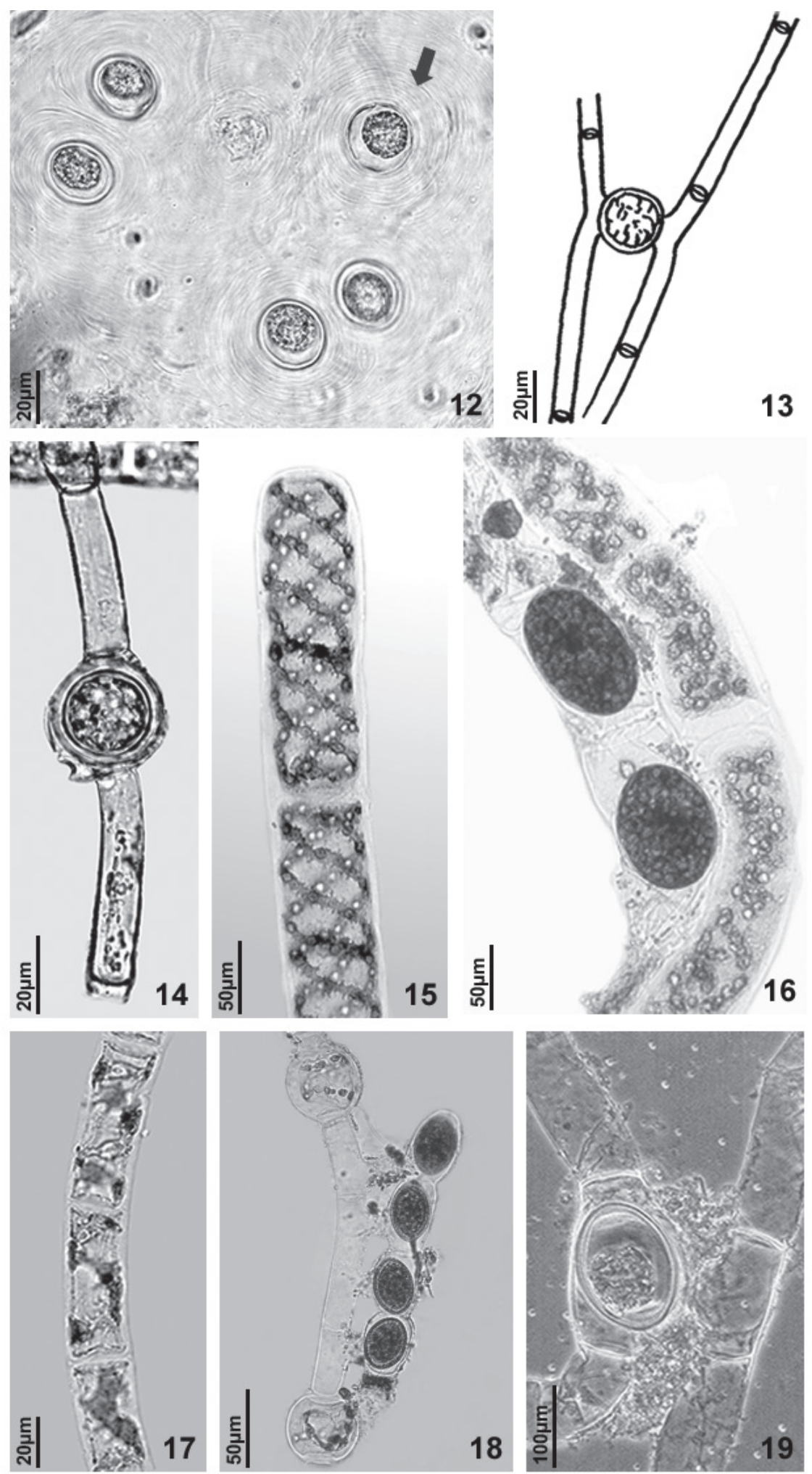

Figuras 12-19. Espécies de macroalgas de nascentes e arredores de riachos na região noroeste do Estado de São Paulo. 12. Gloeocystis cf. ampla, células com bainha concêntrica (seta). 13. Mougeotia gotlandica, parte de filamento em conjugação com zigósporo de paredes enrugadas. 14. Oedogonium globosum f. nanyohense, parte de filamento com oósporo. 15-16. Spirogyra gallica. 15. Parte de filamento em estágio vegetativo. 16. Parte de filamento em conjugação com zigósporos. 17-18. Spirogyra taftiana. 17. Parte de filamento em estágio vegetativo. 18. Parte de filamento em conjugação com zigósporos. 19. Sirocladium robustum, parte de filamento em conjugação com zigósporo.

Figures 12-19. Species of macroalgae in springs and surrounding streams of northwestern region of São Paulo State. 12. Gloeocystis cf. ampla, cells with concentrical sheath (arrow). 13. Mougeotia gotlandica, filament part showing conjugation with wrinkled wall zygospore. 14. Oedogonium globosum f. nanyohense, filament part showing oospore. 15-16. Spirogyra gallica. 15. Filament part in vegetative stage. 16. Filament part showing conjugation with zygospores. 17-18. Spirogyra taftiana. 17. Filament part in vegetative stage. 18. Filament part showing conjugation with zygospores. 19. Sirocladium robustum, filament part showing conjugation with zygospore. 
enquanto os demais foram coletados fora d'água, mas em ambientes úmidos (hábitat subaéreo). Neste contexto, a divisão Chlorophyta foi dominante quanto ao número de espécies (quatro espécies 57\%). Em nascentes, a comunidade algal dominante geralmente é representada pelas Bacillariophyceae (Heterokontophyta), seguida por Chlorophyta, Cyanophyta e Rhodophyta (Whitford 1956, Leake \& Leake 1961, Round 1983, Sherwood et al. 2000, Żelazna-Wieczorek \& Ziułkiewicz 2004). Contudo em nossas análises não foram encontrados representantes das Bacillariophyceae (diatomáceas) que pudessem ser caracterizados como macroalgas.

A ausência de espécies da divisão Rhodophyta talvez possa ser explicada pela limitação da amostragem, já que as coletas foram mensais (fevereiro a setembro de 2007), aleatórias e pontuais, provavelmente subestimando a amostragem deste grupo algal. Além disso, as nascentes amostradas apresentaram condições ambientais que também podem colaborar com a falta de representantes deste grupo. Necchi Júnior et al.(1999) efetuando um estudo sobre a distribuição das Rhodophyta em riachos do Estado de São Paulo, verificaram que estas algas se desenvolveram sob valores médios de condutividade (74 $\mu \mathrm{S} \mathrm{cm}^{-1}$ ) e velocidade da correnteza (48 cm $\mathrm{s}^{-1}$ ), condições estas, completamente distintas das que foram observadas neste estudo (condutividade elétrica de 5,4-19,6 $\mu \mathrm{S} \mathrm{cm}^{-1}$ e velocidade de correnteza praticamente ausente).

Cylindrospermum gorakhpurense, Nostoc muscorum, N. piscinale, Porphyrosiphon notarisii, Gloeocystis cf. ampla e Sirocladium robustum, foram coletadas crescendo entre a vegetação ou solo úmido circundantes às nascentes, confirmando o hábitat tipicamente subaéreo destes organismos (Desikachary 1959, Bicudo \& Picelli-Vicentim 1988, Azevedo 1991, Sant'Anna 1991, Sant'Anna \& Azevedo 1995, Pereira \& Branco 2007).

A flora aquática encontrada diferiu de estudos anteriores nesta região quanto à presença de representantes da família Zygnemataceae em estágio reprodutivo. A maioria destes estudos encontrou numerosos grupos vegetativos (filamentos estéreis) de Spirogyra, Mougeotia e Zygnema (e.g. Branco \& Necchi Júnior 1998, Necchi Júnior et al. 1994, 1997). Contudo, as únicas análises realizadas nesta área que registraram alguma espécie de Zygnemataceae foram as de Necchi Júnior et al. (1994, 1997) que encontraram Spirogyra neglecta (Hassall) Kützing ao avaliar a flora e a distribuição de macroalgas, respectivamente, em 22 e 52 segmentos de riachos.

A taxonomia de categorias infragenéricas de Zygnemataceae baseia-se, essencialmente, em características da fase reprodutiva (reprodução sexuada), complementadas por outras da fase vegetativa (Transeau 1951, Kadlubowska 1984); e devido a este tipo de reprodução sexuada, frequentemente é possível encontrar organismos desta família em estágio reprodutivo em ambientes lênticos (Transeau 1951, Kadlubowska 1984). Dessa maneira, a dominância das Zygnemataceae quanto ao número de espécies nestes ambientes, sugere que as nascentes, devido ao fluxo lento de suas águas, sejam hábitats favoráveis à reprodução destes organismos. Acreditamos ainda, que o fluxo e a oscilação dos níveis d'água nas nascentes permitiram a proliferação e a reprodução de Sirocladium robustum, que é uma espécie tipicamente subaérea, nas imediações deste hábitat.

As espécies Cylindrospermum gorakhpurense R.N. Singh, Mougeotia gotlandica (Cleve) Wittrock, Spirogyra gallica Petit e $S$. taftiana Transeau são registrados pela primeira vez para o país. Tal fato mostra que os estudos envolvendo o conhecimento da flora ficológica deveriam ser permanentes e incentivados, principalmente envolvendo hábitats que muitas vezes são negligenciados pela escassez ou regionalidade de taxonomistas.

\section{Agradecimentos}

Os autores agradecem a Olídio Durão Júnior pelo auxílio nas coletas e Tiago Haruo Ishibashi pela confecção das pranchas de ilustrações.

\section{Literatura citada}

Azevedo, M.T.P. 1991. Edaphic blue-green algae from the São Paulo Botanical Garden, Brasil. Archiv für Hydrobiologie Supplementaband Algological Studies 64: 503-526.

Azevedo, M.T.P., Nogueira, N.M.C. \& Sant'Anna, C.L. 1996. Criptógamos do Parque das Fontes do Ipiranga, São Paulo, SP. Algas, 8: Cyanophyceae. Hoehnea 23: 1-38.

Bicudo, C.E.M. \& Picelli-Vicentim, M.M. 1988. Ficoflórula do campo de esfagno das prateleiras, Parque Nacional de Itatiaia, sul do Brasil. Revista Brasileira de Biologia 48: 15-28.

Borge, O. 1918. Die Von Dr. A. Löfgren in São Paulo gessammelten Süsswasseralgen. Arkiv für Botanik 15: 1-108. 
Branco, C.C.Z. \& Necchi Júnior, O. 1996. Survey of stream macroalgae of eastern Atlantic Rainforest of São Paulo State, southeastern Brazil. Archiv für Hydrobiologie Supplementaband Algological Studies 80: 35-57.

Branco, L.H.Z. \& Necchi Júnior, O. 1998. Distribution of stream macroalgae in three tropical drainage basins of southeastern Brazil. Archiv für Hydrobiologie 142: 241-256.

Branco, L.H.Z., Necchi Júnior, O.\& Branco, C.C.Z. 1999. Cyanophyceae from lotic ecosystems of São Paulo State, Southeastern Brazil. Archiv für Hydrobiologie Supplementaband Algological Studies 94: 63-87.

Branco, L.H.Z. \& Pereira, J.L. 2002. Evaluation of seasonal dynamics and bioindication potential of macroalgal communities in polluted tropical stream. Archiv für Hydrobiologie 155: 147-161.

Branco, L.H.Z., Hoffman, L., Teixeira, J.P., Ferreira, V. \& Morais Filho, J.C. 2009. Aerophytic cyanoprokaryotes from the Atlantic rainforest region of São Paulo State, Brazil: Chroococcales and Oscillatoriales. Cryptogamie, Algologie 30: 135-152.

Desikachary, T.V. 1959. Cyanophyta. Indian Council of Agricultural Research. Monographs on the Algae, New Delhi.

Drouet, F. 1937. The Brazilian Myxophyceae, 1. American Journal of Botany 24: 598-608.

Drouet, F. 1938. The Brazilian Myxophyceae, 2. American Journal of Botany 24: 657-666.

Edwall, G. 1896. Índice das plantas do herbário da Comissão Geográfica e Geológica de São Paulo. Boletim da Comissão geográfica e geológica do Estado de São Paulo 11: 51-215.

Franceschini, I.M. 1983. Levantamento das Nostocophyceae do Rio Seco, Torres, Rio Grande do Sul, Brasil. Dissertação de Mestrado, Universidade Federal do Rio Grande do Sul, Porto Alegre.

Guerrero, P.G. 1948. Nuevos datos ficológicos de España. Anales del Jardín Botánico de Madrid 12: 265-283.

Kadlubowska, J.Z. 1984. Conjugatophyceae I. Chlorophyta VIII: Zygnemales. In: H. Ettl (ed.). Archiv für Hydrobiologie Supplementaband Algological Studies. Gustav Fischer, Jena, pp. 1-532.

Kleerekoper, H. 1944. Introdução ao estudo da Limnologia. Imprensa Nacional, Rio de Janeiro.

Leake, D.V. \& Leake, H.H. 1961. Algae of Ozarkian springs and spring streams: winter aspect near head of Crane Creek, Stone County, Missouri. Proceedings of the Oklahoma Academy of Science 41: 39-46.

Moebius, M. 1895. Ueber einige brasilianische Algen. Hedwigia 34: 173-180.

Necchi Júnior, O. 1992. Macroalgae dynamics in a spring in São Paulo State, southeastern Brazil. Archiv für Hydrobiologie 124: 489-499.

Necchi Júnior, O., Branco, C.C.Z. \& Branco, L.H.Z. 1999. Distribution of Rhodophyta in streams from São Paulo State, southeastern Brazil. Archiv für Hydrobiologie 147: 73-89.

Necchi Júnior, O., Pascoaloto, D., Branco, C.C.Z. \& Branco, L.H.Z. 1994. Distribution of macroalgae in a tropical river basin from southeastern Brazil. Archiv für Hydrobiologie 129: 459-471.

Necchi Júnior, O., Pascoaloto, D., Branco, C.C.Z. \& Branco, L.H.Z. 1997. Stream macroalgal flora of the northwest region of São Paulo State, southeastern Brazil. Archiv für Hydrobiologie Supplementaband Algological Studies 84: 91112.

Oliveira, B.A.D. 1978. Contribuição ao estudo taxonômico das cianofíceas filamentosas da região de São José do Rio Preto, SP. Dissertação de Mestrado, Universidade Federal do Rio de Janeiro, Rio de Janeiro.

Oliveira, B.A.D. \& Cardoso, E.J.B. 1982. Fixação de nitrogênio de algas cianofíceas e sua preservação em laboratório. O Solo 74: 31-35.

Pereira, J.L. \& Branco, L.H.Z. 2006. Espécies do gênero Oedogonium Link em riachos na região noroeste do Estado de São Paulo, Brasil. Anais do XI Congresso Brasileiro de Ficologia e Simpósio Latino-Americano sobre Algas Nocivas. Rio de Janeiro, Museu Nacional, 30: 19-26.

Pereira, J.L. \& Branco, L.H.Z. 2007. Sirocladium robustum, uma nova espécie de Zygnemataceae (Chlorophyta). Hoehnea 34: 103-107.

Randhawa, M.S. 1959. Zygnemataceae. Indian Council of Agricultural Research, New Delhi.

Round, F.E. 1983. Biologia das algas. 2 ed. Guanabara Dois, Rio de Janeiro.

Sant'Anna, C.L. 1988. Scytonemataceae (Cyanophyceae) from the State of São Paulo, southern Brazil. Nova Hedwigia 46: 519-539.

Sant'Anna, C.L. 1991. Two new taxa and Anabaena and other Nostocaceae (Cyanophyceae) from 
the State of São Paulo, southeastern Brazil. Archiv für Hydrobiologie Supplementaband Algological Studies 64: 527-545.

Sant'Anna, C.L. \& Azevedo, M.T.P. 1995. Oscillatoriaceae (Cyanophyceae) from São Paulo State, Brazil. Nova Hedwigia 60: 19-58

Sant'Anna, C.L., Silva, S.M.F. \& Branco, L.H.Z. 1991. Cyanophyceae da Gruta-que-Chora, Município de Ubatuba, Estado de São Paulo, Brasil. Hoehnea 18: 75-97.

Sant'Anna, C.L., Bicudo, R.M. \& Pereira, H.A.S.L. 1983. Nostochophyceae (Cyanophyceae) do Parque Estadual da Ilha do Cardoso, Estado de São Paulo, Brasil. Rickia 10: 1-27.

Sant'Anna, C.L., Pereira, H.A.S.L. \& Bicudo, R.M. 1978. Contribuição ao conhecimento das Cyanophyceae do Parque Estadual das Fontes do Ipiranga, São Paulo, Brasil. Revista Brasileira de Biologia 38: 321-337.

Sheath, R.G. \& Cole, K.M. 1992. Biogeography of stream macroalgae in North America. Journal of Phycology 28: 448-460.

Sherwood, A.R. \& Sheath, R.G. 1999. Seasonality of macroalgae and epilithic diatoms in spring-fed streamsinTexas,USA.Hydrobiologia 390:73-82.

Sherwood, A.R., Rintoul, T.L., Müller, K.M. \& Sheath, R.G. 2000. Seasonality and distribution of epilithic diatoms, macroalgae and macrophytes in a spring-fed stream system in Ontario, Canada. Hydrobiologia 435: 143-152.

Transeau, E.N. 1951. The Zygnemataceae. Ohio State University Press, Columbus.

Vieira Júnior, J., Necchi Júnior, O., Branco, C.C.Z. \& Branco, L.H.Z. 2002. Characeae (Chlorophyta) em ecossistemas lóticos do Estado de São Paulo, Brasil - 1: gênero Nitella. Hoehnea 29: 249-266.
Vis, M.L. \& Sheath, R.G. 1996. Distribution and systematic of Batrachospermum (Batrachospermales, Rhodophyta) in North America. 9. Section Batrachospermum: description of five new species. Phycologia 35: 124-134.

Werner, V.R. 2002. Cyanophyceae/Cyanobacteria no sistema de lagoas e lagunas da planície costeira do estado do Rio Grande do Sul, Brasil. Tese de Doutorado, Universidade Estadual Paulista, Rio Claro.

Werner, V.R. \& Rosa, Z.M. 1992. Cyanophyceae da Estação Ecológica do Taim, Rio Grande do Sul, Brasil. Revista Brasileira de Biologia 52: 481502.

Whitford, L.A. 1956. The communities of algae in the springs and spring streams of Florida. Ecology 37: 433-442.

Whittrock, V.B. \& Nordstedt, C.F.O. 1880. Algae aquae dulcis exsiccate praecipue Scandinaviae, quas adjectis algis marinis chlorophyllaceis et phycochromaceis. Lundae, Typis Berlingiania 7-8: 30-34.

Yunes, J.S. 1990. Cianobactérias fixadoras de nitrogênio do estuário da lagoa dos Patos, RS: Nostoc muscorum. Ciência e Cultura 42: 375383

Yunes, J.S. 1994. Diazotrophic growth and nitrogenase activity of cyanobacteria from the Patos lagoon estuary, southern Brazil. Vittale 6: 25-36.

elazna-Wieczorek, J. \& Ziułkiewicz, M. 2004. Algae communities in springs of Łódź Hills Scarp with diversified hydrochemical conditions. In: PAN. Teka Komisji Ochrony i Kształtowania Środowiska Przyrodniczego, Lublin, v. 1, pp. 322-330. 